\title{
Um Modelo Matemático para Estimativas do Consumo de Energia em Redes de Sensores Visuais sem Fio
}

\author{
M.V.B. CERQUEIRA ${ }^{1 *}$ e D.G. COSTA $^{2}$ \\ Recebido em 16 junho 2018 / Aceito em 22 janeiro 2019
}

\begin{abstract}
RESUMO. As redes de sensores sem fio alcançaram um papel de destaque no cenário atual das novas tecnologias de comunicação, centradas em aplicações de Internet das Coisas e Cidades Inteligentes, sobretudo quando câmeras são utilizadas para obter dados visuais do ambiente monitorado. Nessas redes, questões de eficiência energética são centrais, uma vez que, frequentemente, sensores são alimentados por bateria. Para tanto, um correto e eficiente planejamento energético de redes de sensores sem fio é fundamental para diversas aplicações, levando à escolha mais apropriada dos sensores, dos protocolos de comunicação e das unidades de monitoramento. Assim, visando dar suporte a estimativas de desempenho de redes de sensores visuais sem fio, permitindo análises teóricas sem necessitar da implantação física das redes, este artigo propõe um novo modelo matemático para estimativas de consumo de energia de sensores equipados com câmeras, permitindo importantes avaliações de desempenho antes da implantação de redes reais. Além desse modelo matemático, este artigo também apresenta uma nova ferramenta computacional, EnergyWVSN, desenvolvida para facilitar o uso prático do modelo proposto.
\end{abstract}

Palavras-chave: rede de sensores visuais sem fio, modelagem matemática, consumo de energia, avaliação de desempenho.

\section{INTRODUÇÃO}

As Redes de Sensores Sem Fio (RSSF) são redes formadas por um conjunto de nós sensores autônomos, auto organizáveis e que trocam dados utilizando alguma tecnologia de comunicação sem fio, cujo objetivo primário é obter informações acerca de determinado ambiente de monitoramento [5]. Esses sensores são geralmente pequenos e apresentam capacidades computacionais limitadas, porém possuem recursos suficientes para perceber, medir e coletar informações do meio onde se encontram, transmitindo esses dados para que sejam armazenados, processados ou

\footnotetext{
*Corresponding author: Marcos Vinícius Bião Cerqueira - E-mail: marcosbiao@yahoo.com.br-http://orcid.org/ 0000-0003-1968-6324

${ }^{1}$ PGCA-UEFS, Universidade Estadual de Feira de Santana, Av. Transnordestina, s/n, 44036-900 - Novo Horizonte, Feira de Santana, BA, Brasil. E-mail: marcosbiao@yahoo.com.br

2 DTEC-UEFS, Universidade Estadual de Feira de Santana, Av. Transnordestina, s/n, 44036-900 - Novo Horizonte, Feira de Santana, BA, Brasil. E-mail: danielgcosta@uefs.br
} 
visualizados [27]. Além das limitações computacionais que podem estar presentes, os sensores serão geralmente alimentados por baterias, o que coloca a eficiência energética como um dos pontos centrais ligados ao desempenho dessas redes.

Nos últimos anos, as RSSF vêm ganhando muito espaço devido a sua flexibilidade de aplicações [14], podendo ser utilizadas em diversas áreas [10]. Nos cenários complexos em formação, sobretudo em aplicações na Internet das Coisas e nas Cidades Inteligentes [19,22], as redes de sensores sem fio podem ser utilizadas para fornecer informações valiosas do funcionamento de sistemas paralelos e do comportamento das pessoas, contribuindo para as transformações previstas para este século.

Com o melhoramento dos recursos presentes nos sensores e com a redução dos custos de aquisição, câmeras de baixo consumo energético puderam ser acopladas para capturar imagens e vídeos, permitindo o surgimento das Redes de Sensores Visuais Sem Fio (RSVSF). Com isso, aumentaram-se as possibilidades de aplicações, sobretudo nas áreas de vigilância, rastreamento e localização, abrindo uma nova fronteira de pesquisa e desenvolvimento [3,26]. Contudo, a transmissão de dados visuais requer uma maior banda de comunicação do que a transmissão de dados escalares, além de demandar maior processamento em suas ações, potencialmente aumentando o consumo energético [4]. No geral, as RSVSF serão mais complexas que as redes de sensores sem fio de dados escalares, o que aumenta ainda mais a necessidade por técnicas eficientes de avaliação de desempenho.

De modo geral, a transmissão de dados visuais (imagens e/ou vídeos) requer uma grande quantidade de recursos, se comparada com a realidade dos nós sensores que transmitem dados escalares (temperatura, pressão, umidade, etc). Com isso, o desempenho energético deve ser prioritário no desenvolvimento dos projetos, levando em consideração que o tempo operacional das redes é proporcional à quantidade de energia remanescente nos seus nós.

Para propor métodos que possam minimizar o gasto de energia em RSSF e RSVSF, é preciso compreender com exatidão as características do consumo energético nos elementos que compõe a rede considerada. Dessa forma, conhecendo os detalhes envolvidos nos processos de captura, processamento e transmissão, é possível desenvolver modelos matemáticos que possam estimar, com certa precisão, o consumo de energia de um nó sensor [21].

Existem algumas formas de validar um projeto de rede de sensores. Uma dessas formas é implantar uma rede real em escala reduzida. No geral, esse método pode trazer resultados confiáveis, mas implementar uma rede, apenas para validação, pode ser um desperdício de recursos, levando em consideração o material necessário, as pessoas envolvidas e o tempo para testes. Por outro lado, modelos matemáticos apresentam-se como uma solução viável para validação de redes, uma vez que com apenas um computador relativamente simples em termos de capacidade de processamento é possível realizar testes de forma rápida, modificando apropriadamente os parâmetros de configuração.

O consumo de energia é um dos pontos mais críticos em uma rede de sensores sem fio, uma vez que está intimamente ligado ao tempo de vida da rede. Assim, estimar o consumo de energia é 
uma parte importante do ciclo de vida da rede e alguns trabalhos na literatura propõe modelos para esse fim. De fato, alguns modelos matemáticos vêm sendo propostos para redes de sensores sem fio, porém nenhum modelo foi desenvolvido especificamente para redes de sensores visuais. Embora possam existir adaptações de modelos de RSSF para emular sensores visuais, os resultados se distanciam de uma situação real devido às aproximações. Para que os resultados sejam mais satisfatórios é necessário desenvolver um modelo próprio para sensores visuais sem fio.

Esse artigo apresenta um novo modelo matemático parametrizável e unificado para sensores visuais, permitindo estimativas de consumo energético de nós sensores equipados com câmera. Tais estimativas estarão centradas nos processos de captura dos dados, codificação, criptografia e "paquetização" (criação de pacotes de dados para serem enviados pela rede). De fato, embora as etapas relacionadas à transmissão dos dados não sejam modeladas, uma vez que podem ser mapeadas para qualquer modelo de transmissão já existente, o modelo matemático proposto é bastante necessário, uma vez que permite medir o impacto energético do processamento realizado pelos nós sensores visuais que capturam e codificam dados visuais (nós fontes). Assim, pesquisadores e profissionais poderão avaliar o perfil de consumo de energia de nós fontes para diferentes configurações, auxiliando nas etapas de projeto e gerenciamento da rede.

Este artigo está organizado da seguinte forma. A Seção 2 apresenta trabalhos relacionados presentes na literatura. O modelo proposto é descrito na Seção 3. A Seção 4 apresenta inúmeros resultados de verificação matemática em cenários de testes, seguida pelas conclusões e referências do artigo.

\section{TRABALHOS RELACIONADOS}

Em geral, espera-se que o processamento e a transmissão de dados visuais tenham maior custo computacional, seja por conta da captura e processamento das informações ou por transmissão das mesmas, gerando um maior gasto energético [4]. Portanto, análises de desempenho relacionadas ao consumo de energia são bastante necessárias. De fato, na literatura, é possível encontrar diversos trabalhos que propõe ou utilizam alguma modelagem de energia no contexto das redes de sensores sem fio, influenciando este artigo de diferentes formas.

No trabalho descrito em [15] é realizado um estudo a respeito do consumo de energia na codificação de imagens no popular formato JPEG (Joint Photographic Experts Group). Para tanto, foi realizada uma simplificação do modelo de consumo de energia para cada etapa do processo de codificação, permitindo assim estimativas da quantidade de energia dissipada pelo nó sensor. Essa simplificação foi desenvolvida baseando-se nas operações básicas realizadas pelo método de codificação de imagem utilizado (JPEG). O JPEG é um algoritmo de codificação de imagens popular na Internet e que é centrado na codificação DCT (Discrete Cosine Transform), uma transformada de cosseno bastante usada para processamento de sinais.

Em [12] é estudado um esquema de codificação DWT (Discrete Wavelet Transform), onde é apresentado o modelo de consumo de energia do algoritmo de codificação JPEG2000. É apresentada também uma tabela com diversos parâmetros da plataforma de desenvolvimento embar- 
cado "Mica2", que é empregado para validação do método. Por fim, foram apresentadas algumas tabelas com os resultados de execuções utilizando a codificação DWT, enfatizando a economia de energia obtida.

Em [25] é feita uma comparação de dois algoritmos de criptografia de chave pública, o RSA ( $R i$ vest-Shamir-Adleman), baseado na fatoração de números primos, e o ECC (Elliptic Curve Cryptografy), baseado em curvas elípticas. Os autores fazem uma análise acerca do gasto energético em ambos os algoritmos, detalhando a quantidade de energia consumida no processo de assinatura e de verificação. É realizada também análises do consumo de energia para o algoritmo de criptografia simétrica AES-128 e para o algoritmo de função hash SHA-1.

O artigo [7] concentra-se na análise do algoritmo de criptografia simétrica AES (Advanced Encryption Standard). Nele é exposto o modelo de consumo de energia, o qual é baseado nas instruções (and, or e shift) necessárias para realizar a maior parte do processamento e que diretamente impactam no consumo de energia. É comparada também a diferença de consumo quanto ao tamanho da chave criptográfica utilizada (128 bits, 192 bits e 256 bits).

Em [20] foi proposto um modelo para estimar o consumo de energia na unidade de processamento de um nó sensor. Nesse trabalho foram desenvolvidos dois modelos, sendo um baseado em Cadeia de Markov e outro em Rede de Petri. Grosso modo, uma Cadeia de Markov é utilizada para modelar processos estocásticos (não determinísticos) através de estados discretos. Já as Redes de Petri são utilizadas para modelar sistemas discretos na forma de gráficos direcionados. Ambos os formalismos são utilizados em [20] para medir o consumo de energia na unidade central de processamento de sensores. Nas simulações realizadas na ferramenta "MatLab", a Rede de Petri mostrou-se mais eficiente do que a Cadeia de Markov, como ferramenta de modelagem. De fato, Redes de Petri são uma importante ferramenta formal para modelagem de sistemas discretos, sendo utilizada também na especificação do modelo proposto neste trabalho.

Em [1] foi utilizada uma modelagem em Rede de Petri Colorida para avaliar um protocolo de controle de acesso, permitindo maiores níveis de abstração. Como a Rede de Petri Colorida é hierárquica, ela permite a construção de um grande módulo como um conjunto de pequenos módulos, uns ligados aos outros, permitindo, assim, redução de um modelo mais complexo em partes menores. Após a construção do modelo, podem-se extrair medidas de desempenho (consumo de energia e latência) utilizando simulações.

O trabalho descrito em [6] apresenta uma abordagem para o desenvolvimento de aplicações móveis real-time para localização e monitoramento em ambientes fechados. Sendo um sistema de tempo real, as exigências por mecanismos mais eficientes de processamento e transmissão são ainda maiores, estimulando o uso de modelos formais de modelagem. De fato, nesse trabalho utilizou-se uma Rede de Petri Colorida para modelagem do comportamento do sistema.

O trabalho [16] utiliza Redes de Petri para realizar estimativas de consumo de energia de sensores escalares tradicionais, sem uso de câmera. Nesse trabalho, a Rede de Petri projetada consegue simular a tendência do consumo de energia da rede de sensores analisada, obtendo resultados próximos de medições reais. 
De fato, a utilização de Redes de Petri para medições e estimativas de consumo de energia em redes de sensores tem apresentado resultados promissores, sendo uma abordagem viável para diversos cenários.

Além de Redes de Petri, ferramentas computacionais podem ser desenvolvidas para estimar o consumo de energia em redes de sensores, desde que implementem um modelo matemático consistente para embasar os cálculos realizados, como visto em [8,9,28]. Ferramentas computacionais para estimativas de desempenho são muito úteis pois facilitam sobremaneira diversos tipos de análises, uma vez que as ferramentas são geralmente desenvolvidas sobre um modelo matemático já definido. Assim, não é preciso criar um modelo específico. Além disso, a construção de ferramentas permite considerar aspectos significativos que não são observados por métodos habituais encontrados na literatura, deixando mais confiável todo o processo de análise.

Um tipo especial de ferramenta de análise de desempenho são os simuladores. Usando modelos matemáticos como base, os simuladores tentam emular a operação de uma rede ao longo do tempo, com resultados satisfatórios em grande parte dos casos. Simuladores como Castalia, NS3 e Shawn conseguem modelar uma determinada rede e verificar o desempenho de comunicação, o que inclui cobertura, taxas de erros e consumo de energia. Trabalhos como $[13,17,24]$ utilizam simuladores para comparar protocolos e validar suas eficiências energéticas. Apesar de promissores, contudo, a literatura ainda carece de métodos que considerem aspectos de hardware para realizar estimativas mais realísticas, além das dificuldades e simplificações encontradas quando sensores visuais são empregados (a maior parte dos simuladores são destinados à redes de sensores escalares).

Nesse contexto, é possível notar a existência de diferentes recursos para validação e análise de desempenho de rede de sensores. De fato, cada recurso possui vantagens e desvantagens que devem ser avaliadas com atenção. Contudo, quando se trata de redes de sensores visuais sem fio e do processamento nos nós sensores, não existem recursos de verificação satisfatórios. $\mathrm{O}$ modelo proposto neste trabalho e a ferramenta desenvolvida apresentam-se, portanto, como um efetivo recurso para atender essa demanda.

\section{MODELO DESENVOLVIDO}

O objetivo deste artigo é propor um modelo matemático parametrizável para estimativas de desempenho em Redes de Sensores Visuais Sem Fio. O modelo a ser proposto possui como característica central o fato de não necessitar de alto poder computacional e por ser possível realizar testes de forma rápida, potencialmente beneficiando pesquisas na área.

O modelo proposto aborda uma parte importante do processo operacional das redes de sensores visuais sem fio, que é o processamento interno nos nós sensores. De fato, foram realizadas algumas simplificações a fim de tornar o modelo factível, porém, ainda mantendo similaridade com a forma como ocorrem efetivamente os processos modelados. A Figura 1 apresenta uma visão macro do modelo global na totalidade, detalhando o papel do modelo proposto, uma vez que a modelagem do processo de comunicação entre os nós sensores foi deixada para trabalhos futu- 
ros. Como a comunicação em geral envolve processos não determinísticos, outras modelagens são necessárias, porém, o modelo proposto se adequaria perfeitamente uma vez que a modelagem é modularizada. Em outras palavras, espera-se que a modelagem proposta neste trabalho seja um primeiro passo para a modelagem matemática completa de redes de sensores visuais sem fio.

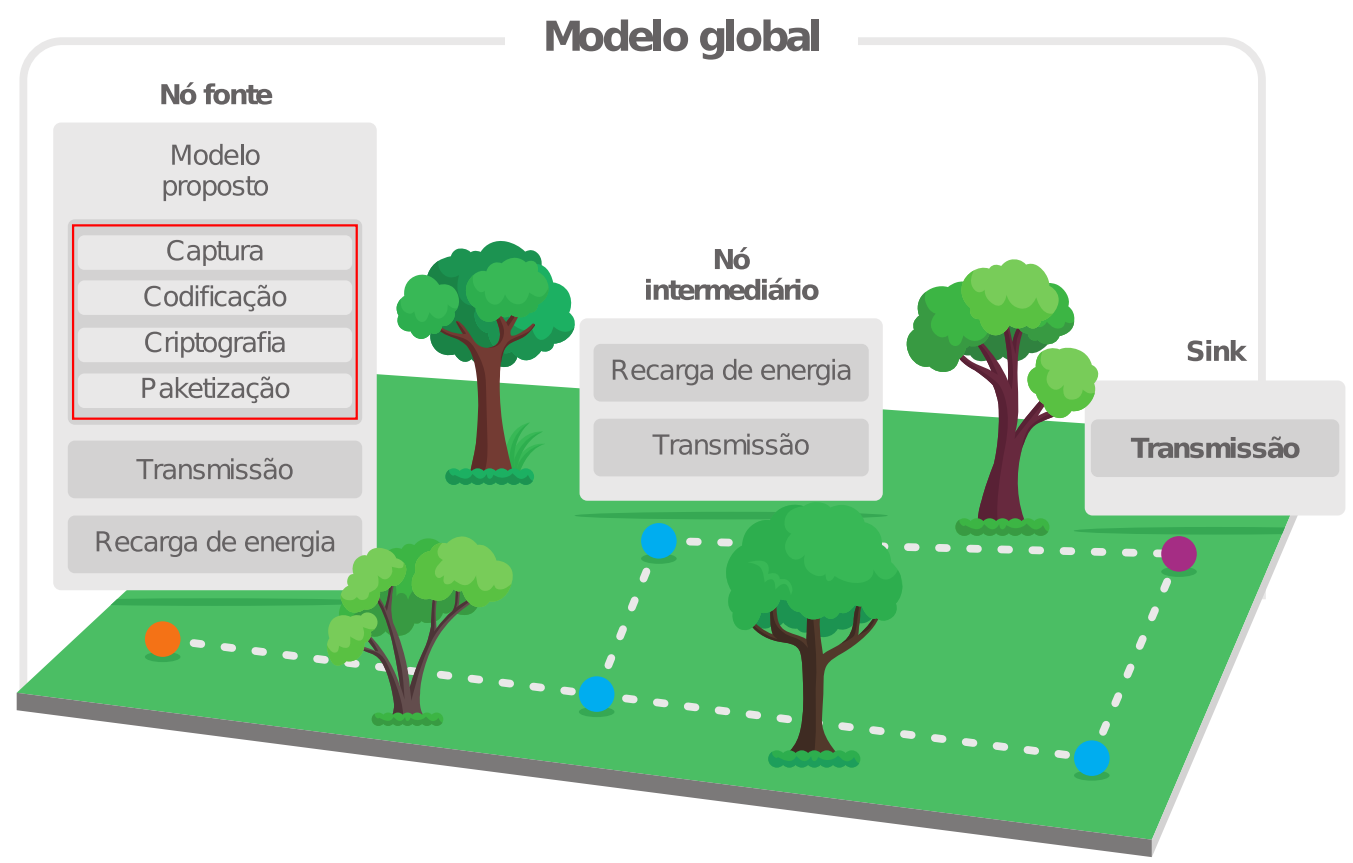

Figura 1: Uma perspectiva global para a modelagem matemática do consumo de energia em RSVSF.

O modelo global idealizado deve ser capaz de lidar com todos os aspectos relacionados às redes de sensores visuais sem fio, no tocante ao consumo de energia. Assim, além das questões relacionadas à captura, codificação, criptografia e paquetização, um modelo global deve também contemplar estimativas para a transmissão de dados pela rede e questões relacionadas à recarga de energia.

De fato, a fim de permitir sua utilização prática sem perdas de generalidade, o modelo proposto apresenta as seguintes simplificações:

- Apenas os nós fontes são modelados, uma vez que esse tipo de nó consome parte significativa da energia da rede;

- Somente capturas de imagens foram modeladas, deixando o processamento de vídeo para trabalhos futuros;

- Capturas são realizadas periodicamente, seguindo o comportamento definido para os sensores. Assim, não foi modelada a captura de dados em resposta à requisições externas. 
Com o intuito de facilitar o desenvolvimento e utilização prática em processos de avaliação de desempenho, o modelo proposto está divido em módulos de acordo com as funcionalidades esperadas de um nó da rede, sempre com entradas e saídas bem definidas. Dessa forma, é possível que o usuário do modelo proposto possa utilizar apenas os módulos desejados para verificações de consumo de energia.

A lista de módulos definidos neste trabalho é apresentada a seguir:

- Captura: responsável por estimar o consumo de energia no processo de captura de imagens;

- Codificação: responsável por estimar o consumo de energia no processo de codificação de imagens;

- Criptografia: responsável por estimar o consumo de energia na encriptação e decrepitação dos dados;

- Paquetização: responsável por estimar o consumo de energia no processo de divisão de dados em pacotes.

Os módulos são unidades lógicas que agrupam equações que definem ações esperadas de um nó sensor na rede. Sendo assim, os módulos possuem uma sequência lógica pré-definida, não podendo ser alterada, como apresentado na Figura 2. Os módulos de codificação e de criptografia são de uso opcional, podendo ser retirados da execução do modelo de acordo com as características da rede que está sendo modelada. As saídas com as quantidades de energia serão somadas para estimar o consumo total de energia de determinado nó.

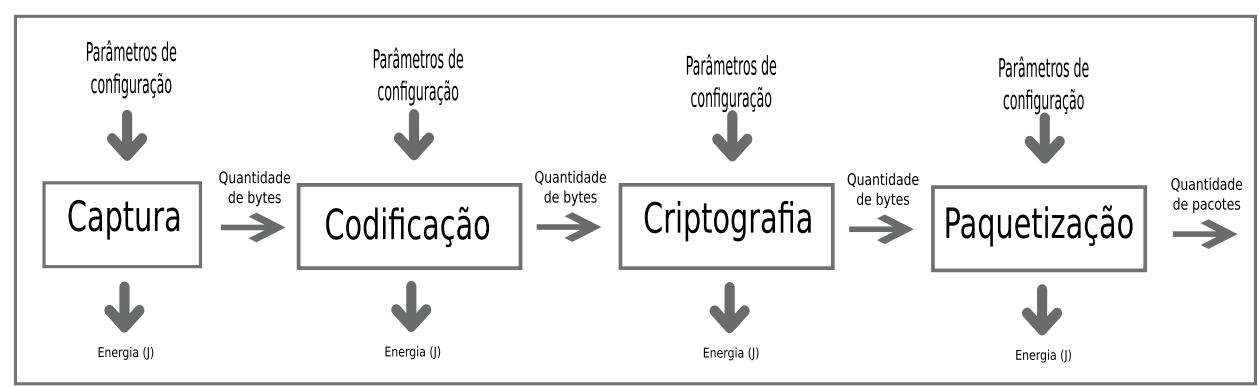

Figura 2: Modelo genérico proposto para entrada e saída de dados.

Além das equações de consumo de energia, presentes em cada módulo, a solução proposta foi modelada utilizando o formalismo de Rede de Petri [2], garantindo a inexistência de deadlocks. De maneira geral, deadlocks ocorrem quando existe um impasse entre dois ou mais processos, levando a um "bloqueio" no processamento. A utilização de Redes de Petri permite então que 
determinado sistema discreto possa ser verificado em relação à presença de deadlocks. A Figura 3 apresenta o diagrama geral da Rede de Petri criada para o modelo de consumo de energia proposto.

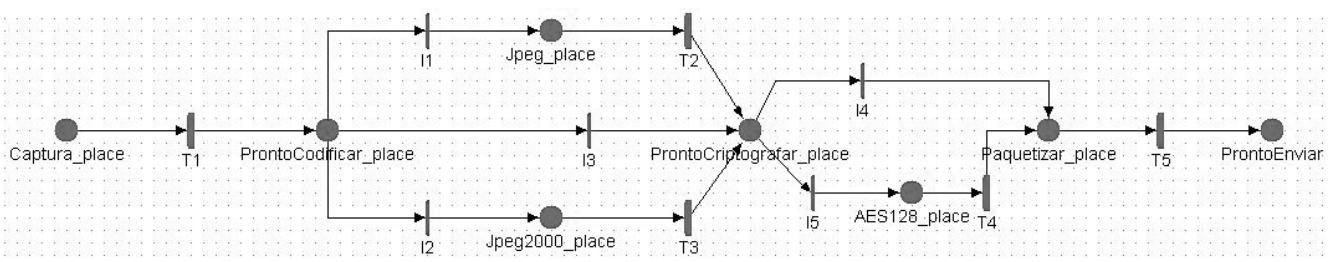

Figura 3: Rede de Petri do modelo proposto.

As propriedades da Rede de Petri são verificadas inspecionando-se o grafo em questão. Com isso, observam-se, além das propriedades básicas, os fluxos na rede que possam causar situações críticas como deadlocks. Essa análise é de extrema importância, pois garante que o sistema nunca entrará em uma conjuntura na qual não existirá um estado subsequente. No caso de existir alguma possibilidade dessas situações, é necessário definir critérios para corrigir o fluxo da rede.

Com a verificação da Rede de Petri, pode-se afirmar que situações como as citadas anteriormente não ocorrerão na rede apresentada, uma vez que foi realizada uma análise dos fluxos presentes na rede, seis ao total, os quais não apresentaram tais situações. Portanto, a propriedade de vivacidade é atendida, evidenciando a falta de inconsistências no modelo proposto.

Portanto, o modelo apresentado será útil para projetos de diversos pesquisadores, podendo ser utilizado para validação do tempo de vida útil da rede. Adicionalmente, espera-se que o modelo proposto permita compreender, de forma mais precisa, o perfil de consumo energético dos nós sensores visuais sem fio.

\subsection{Modelagem do consumo de energia}

De maneira geral, cada módulo possui três componentes lógicos, como apresentado a seguir:

- Parâmetros de configuração: informações definidas pelo usuário do módulo, referentes a ajustes do ambiente de verificação;

- Entrada do módulo: representa os dados que são inseridos para processamento provenientes de outro módulo. O módulo de captura não possui o componente "entrada";

- Saída do módulo: representa os dados que são fornecidos a outro módulo ou mesmo a saída final do sistema modelado (nó sensor fonte).

Os quatro módulos desenvolvidos são descritos nas próximas subseções. 


\subsection{Módulo de captura}

O módulo de captura é encarregado de calcular a quantidade de energia despendida no processo de captura de informações e a quantidade de bytes gerados nessa captura.

A quantidade de bytes gerados é de suma importância para os módulos subsequentes, porque eles se baseiam na quantidade de dados que será processada para calcular a energia que será consumida no processo. A Tabela 1 apresenta os componentes lógicos do módulo de captura.

Tabela 1: Módulo de captura.

\begin{tabular}{ll}
\hline \multicolumn{1}{c}{ Símbolo } & \multicolumn{1}{c}{ Parâmetros de configuração } \\
\hline$c c$ & \multicolumn{1}{c}{ Item } \\
\hline$c o$ & Cuantidade de energia consumida pela câmera para processar 1 byte (J) \\
\hline$f r$ & Frequência de captura (capturas/minuto) \\
\hline$r$ & Resolução da câmera (pixels) \\
\hline$t s$ & Tempo de simulação (hora) \\
\hline \multicolumn{1}{c}{ Símbolo } & \multicolumn{1}{c}{ Saída } \\
\hline$Q s$ & Quantidade de bytes produzidos nesse módulo (será a entrada de dados \\
& do módulo seguinte) \\
\hline$E m_{(c)}$ & Quantidade de energia consumida pelo processo de captura das \\
& informações \\
\hline
\end{tabular}

\section{Equações}

\begin{tabular}{ll}
\hline \multicolumn{1}{c}{ Fórmula } & \multicolumn{1}{c}{ Descrição } \\
\hline$q t d=(t s * 60) * f r$ & Quantidade de capturas realizadas em todo processo \\
\hline$Q s=r * c o$ & Quantidade de bytes gerados em 1 captura \\
\hline$E m_{c}=Q s * c c$ & Energia consumida em 1 captura \\
\hline$E m_{c}=\sum_{i=1}^{q t d} E m_{c}(i)$ & Energia consumida em todo processo de captura \\
\hline
\end{tabular}

\subsection{Módulo de codificação}

Esse módulo é responsável por calcular a quantidade de energia consumida no processo de codificação da imagem. Esse é um módulo opcional, pois, é possível transmitir a imagem original, não a convertendo em outro formato. Tem-se como base a quantidade de bytes e a quantidade de pixels da imagem original. A Tabela 2 apresenta os componentes lógicos do módulo de codificação. As equações presentes neste módulo são apresentadas em [11, 12, 15, 23], sendo re-escritas e formatadas neste artigo.

Como o Módulo de Codificação prevê cálculos para codificação DCT e DWT, espera-se a utilização dos populares codecs de imagem JPEG e JPEG2000. Além disso, deve-se notar que o consumo de energia apresentado é em Joules (J). 
Tabela 2: Módulo de codificação.

\begin{tabular}{|c|c|c|}
\hline \multicolumn{3}{|c|}{ Parâmetros de configuração } \\
\hline Símbolo & & Item \\
\hline$\varepsilon_{\text {add }}$ & \multicolumn{2}{|c|}{ Energia consumida pelo nó sensor com instrução de adição } \\
\hline$\varepsilon_{\text {mult }}$ & \multicolumn{2}{|c|}{ Energia consumida pelo nó sensor com instrução de multiplicação } \\
\hline$\varepsilon_{\text {div }}$ & \multicolumn{2}{|c|}{ Energia consumida pelo nó sensor com instrução de divisão } \\
\hline$\varepsilon_{r}$ & \multicolumn{2}{|c|}{ Energia consumida pelo nó sensor com instrução de round } \\
\hline$\varepsilon_{\text {shift }}$ & \multicolumn{2}{|c|}{ Energia consumida pelo nó sensor com o processo de troca de bits } \\
\hline$\varepsilon_{\text {rmem }}$ & \multicolumn{2}{|c|}{ Energia consumida para ler 1 byte da memória flash } \\
\hline$\varepsilon_{\text {wmem }}$ & \multicolumn{2}{|c|}{ Energia consumida para escrever 1 byte na memória flash } \\
\hline \multicolumn{3}{|c|}{ Entrada } \\
\hline Símbolo & \multicolumn{2}{|r|}{ Item } \\
\hline$Q b$ & \multicolumn{2}{|c|}{ Quantidade de bytes gerada no módulo anterior } \\
\hline \multicolumn{3}{|r|}{ Saída } \\
\hline Símbolo & \multicolumn{2}{|r|}{ Item } \\
\hline Qs & \multicolumn{2}{|c|}{$\begin{array}{l}\text { Quantidade de bytes produzidos nesse módulo (será a entrada de dados } \\
\text { do módulo seguinte) }\end{array}$} \\
\hline$E m_{(c)}$ & \multicolumn{2}{|c|}{$\begin{array}{l}\text { Quantidade de energia consumida pelo processo de captura das } \\
\text { informações }\end{array}$} \\
\hline \multicolumn{3}{|c|}{ Equações } \\
\hline \multicolumn{3}{|c|}{$\begin{array}{ll}\text { Fórmula } & \text { Descrição }\end{array}$} \\
\hline \multicolumn{3}{|c|}{$\begin{array}{lllll}E_{p}=E_{d c t}+E_{q}+E_{z}+E_{\text {rle }}+E_{\text {huf }} & \begin{array}{l}\text { Quantidade de energia consumida pela } \\
\text { codificação DCT }\end{array} & & \\
\end{array}$} \\
\hline \multicolumn{3}{|c|}{$\begin{array}{l}E_{d c t}=\left(\frac{N}{k}\right)^{2} 2 k^{2}\left(k \varepsilon_{m u l t}+(k-\text { Energia consumida com a transformada 2D DCT }\right. \\
\left.1) \varepsilon_{\text {add }}\right)\end{array}$} \\
\hline \multicolumn{2}{|c|}{$E_{q}=\left(\frac{N}{k}\right)^{2} 2 k^{2}\left(\varepsilon_{d i v}+\varepsilon_{r}\right)$} & Energia consumida na etapa de Quantização \\
\hline \multicolumn{2}{|c|}{$E_{z}=\left(\frac{N}{k}\right)^{2}\left(k^{2}-1\right) \varepsilon_{s h}$} & Energia consumida na etapa de zigzag \\
\hline \multicolumn{3}{|c|}{$E_{r l e}=\sum_{j=1}^{\left(\frac{N}{k}\right)^{2}} \varepsilon_{r l e} \quad$ Energia consumida na etapa de RLE } \\
\hline \multicolumn{3}{|c|}{$E_{h u f}=\sum_{j=1}^{\left(\frac{N}{k}\right)^{2}} \varepsilon_{h u f_{j}^{D C}}+\varepsilon_{h u f_{j}^{A C}}$} \\
\hline $\begin{array}{l}E_{d w t}(M, N, T \\
12 \varepsilon_{\text {add }} \\
\left.2 \varepsilon_{w m e m}\right) \sum_{i=}^{T}\end{array}$ & $\begin{array}{cc}=M N\left(10 \varepsilon_{\text {shift }}+\right. \\
2 \varepsilon_{\text {rmem }}+ \\
\end{array}$ & Energia consumida pela codificação DWT \\
\hline
\end{tabular}

\subsection{Módulo de criptografia}

Este é um módulo opcional e se encarrega de calcular a quantidade de energia consumida no processo de encriptar as informações. Para tanto, tem-se como base a quantidade de bytes gerados nos módulos anteriores para realizar esse procedimento. Neste trabalho, utilizou-se apenas 
criptografia simétrica, uma vez que essa é uma abordagem comum para redes de sensores visuais sem fio.

A Tabela 3 apresenta os componentes lógicos do Módulo de Criptografia. As equações presentes neste módulo são baseadas nos trabalhos apresentados em [7, 18].

Tabela 3: Módulo de criptografia.

\begin{tabular}{|c|c|c|}
\hline \multicolumn{3}{|c|}{ Parâmetros de configuração } \\
\hline Símbolo & & Item \\
\hline TipoCripto & Indicação de qual c & riptografia usar \\
\hline$\varepsilon_{\text {shift }}$ & Quantidade de ener & gia consumida por uma instrução shift \\
\hline$\varepsilon_{\text {and }}$ & Quantidade de ener & gia consumida por uma instrução and \\
\hline$\varepsilon_{\text {or }}$ & Quantidade de ener & gia consumida por uma instrução $o r$ \\
\hline$n_{r}$ & Número de interaçć & es por bloco \\
\hline$n_{b}$ & Tamanho do bloco & utilizado \\
\hline \multicolumn{3}{|r|}{ Entrada } \\
\hline Símbolo & & Item \\
\hline$Q b$ & Quantidade de byte & s gerada no módulo anterior \\
\hline \multicolumn{3}{|c|}{ Saída } \\
\hline Símbolo & & Item \\
\hline Qs & $\begin{array}{l}\text { Quantidade de byte } \\
\text { do módulo seguinte }\end{array}$ & $\begin{array}{l}\text { s produzidos nesse módulo (será a entrada de dados } \\
\text { ) }\end{array}$ \\
\hline$E m_{(c)}$ & $\begin{array}{l}\text { Quantidade de en } \\
\text { informações }\end{array}$ & ergia consumida pelo processo de captura das \\
\hline \multicolumn{3}{|r|}{ Equações } \\
\hline \multicolumn{3}{|c|}{ Símbolo } \\
\hline \multicolumn{3}{|c|}{$\begin{array}{l}E m_{y}=\left\{\left(46 n_{b} n_{r}-30 n_{b}\right) \varepsilon_{\text {and }}+\text { Energia consumida no processo de criptografia }\right. \\
{\left[31 n_{b} n_{r}+12\left(n_{r}-1\right)-\text { AES-128 }\right.} \\
\left.20 n_{b}\right] \varepsilon_{o r}+\left[64 n_{b} n_{r}+96\left(n_{r}-\right.\right. \\
\left.\left.1)-61 n_{b}\right] n_{\text {shift }}\right\} n b l\end{array}$} \\
\hline$n b l=Q b / 16$ & & Quantidade de interações \\
\hline
\end{tabular}

\subsection{Módulo de paquetização}

O módulo de paquetização é encarregado de calcular a quantidade de energia despendida no processo de divisão da imagem a ser transmitida em pacotes.

Apesar do modelo não se preocupar com o processo de transmissão de dados, este Módulo considera que a divisão dos dados em pacotes é de responsabilidade do nó fonte, que é o elemento em foco deste trabalho. A Tabela 4 apresenta os componentes lógicos para o Módulo de Paquetização. 
Tabela 4: Módulo de paquetização.

\begin{tabular}{|c|c|}
\hline \multicolumn{2}{|r|}{ Parâmetros de configuração } \\
\hline Símbolo & Item \\
\hline Recc & da imagem \\
\hline Tam & do pacote \\
\hline $\mathrm{Cab}$ & pacote \\
\hline \multicolumn{2}{|r|}{ Entrada } \\
\hline Símbolo & Item \\
\hline Qual & bytes gerada no módulo anterior \\
\hline \multicolumn{2}{|r|}{ Saída } \\
\hline Símbolo & Item \\
\hline Núm & de pacotes gerados \\
\hline $\begin{array}{l}\text { Quat } \\
\text { em p }\end{array}$ & energia consumida pelo processo de divisão dos dados \\
\hline \multicolumn{2}{|r|}{ Equações } \\
\hline Símbolo & Item \\
\hline$n p=\left|\frac{(b+o)}{K-z}\right|$ & $\begin{array}{l}\text { Quantidade de pacotes necessários para transmitir a } \\
\text { imagem }\end{array}$ \\
\hline$u p=\left(\frac{b+o}{K-z}-n p\right) * K$ & Tamanho do último pacote $K$ \\
\hline$t b=n p * K+u p$ & Quantidade total de bytes a ser transmitido \\
\hline$E m_{(p)}=t b * \varepsilon_{w m e m}$ & $\begin{array}{l}\text { Energia consumida para gravar os pacotes na memória } \\
\text { RAM }\end{array}$ \\
\hline
\end{tabular}

A saída do Módulo de Paquetização difere um pouco dos módulos anteriores. Até então, tinhase como saída uma determinada quantidade de bytes, a qual é utilizada nos módulos seguintes para realizar seus cálculos. Porém este módulo tem como saída a quantidade de pacotes a ser transmitida, já que o módulo de transmissão, idealizado em um modelo global, utiliza os pacotes prontos, em vez de manipular quantidades de bytes.

\subsection{Ferramenta para verificação do modelo}

Para realizar os testes do modelo desenvolvido e, consequentemente, analisar os resultados, foi desenvolvida uma ferramenta que, de forma intuitiva, facilita a utilização do modelo proposto. Essa ferramenta, EnergyWVSN, foi projetada para ser prática e fácil de usar. A Figura 4 apresenta uma tela inicial da ferramenta, demonstrando que é possível configurar todos os aspectos referentes ao modelo apresentado. Nessa etapa, deve-se configurar as 4 opções apresentadas.

A primeira opção de configuração disponível é “Sensor”. Nela deve-se escolher qual o sensor será utilizado. A ferramenta traz consigo dois sensores já tabelados, o Mica2 e o Raspberry Pi 2, mas 


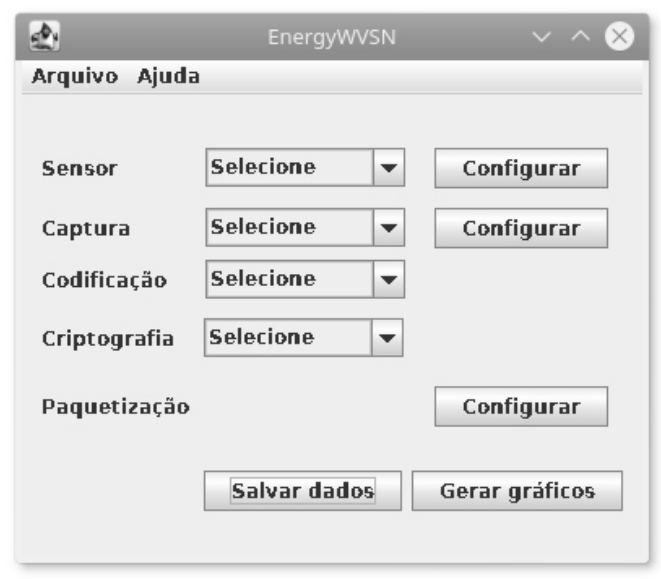

Figura 4: Tela principal da ferramenta.

o usuário pode optar em utilizar um sensor diferente, contanto que informe o valor de consumo de energia para as ações do sensor, garantindo flexibilidade na utilização da ferramenta.

A segunda opção é Captura. Nessa opção, deve-se configurar os aspectos relacionados à câmera utilizada e às imagens a serem capturadas. A ferramenta traz consigo duas câmeras tabeladas, mas é possível configurar outras câmeras. Além da câmera, é necessário ajustar aspectos importantes da captura de imagens por sensores visuais, como resolução, frequência de captura e coloração da imagem.

Além dessas configurações, é possível utilizar os módulos de Codificação e de Criptografia, que são opcionais. Quanto a Codificação, pode-se optar por usar JPEG, JPEG2000 ou não utilizar codificação. Já para criptografia, opta-se por usar AES-128 ou não usar criptografia. A última opção refere-se ao módulo de paquetização, o qual se configuram os parâmetros referentes a divisão dos dados em pacotes. Todas essas opções estão disponíveis na versão inicial da ferramenta, porém novas configurações estarão disponíveis em versões posteriores.

Há ainda opções de suporte na ferramenta, ligadas ao salvamento e abertura de configurações pre-estabelecidas, facilitando a adoção da mesma para verificações de redes de sensores visuais com diferentes configurações.

\section{RESULTADOS}

A fim de validar o modelo matemático proposto, uma série de verificações foram realizadas. De fato, a realização dos testes ocorreu de duas formas diferentes. No primeiro teste a ferramenta foi executada com o objetivo de verificar o resultado emitido pela mesma, não realizando nenhuma comparação. O segundo teste tinha como objetivo verificar se o modelo possui exatidão suficiente para estimar o consumo de energia. Para tal, foram comparados os resultados obtidos por este 
modelo com uma medição realizada em uma situação real, utilizando a plataforma Raspberry Pi 2 para efetuar essa medição.

\subsection{Usando a ferramenta}

Para a realização do primeiro grupo de testes, utilizaram-se as seguintes configurações como referência:

- Sensor: Mica 2

- Câmera: OV7670

- Tempo de execução: 1 hora

- Frequência de captura: 10 imagens por minuto

- Resolução das imagens: 500 x 500

- Coloração: Colorido

- Codificação: JPEG2000

- Criptografia: AES128

- Tamanho do pacote: 50 bytes

- Tamanho do cabeçalho: 5 bytes

- Tamanho do reconstrutor: 4 bytes

Ao utilizar a opção "Gerar gráficos", têm-se os resultados apresentados na Figura 5, que são apenas parte dos resultados possíveis com a ferramenta.

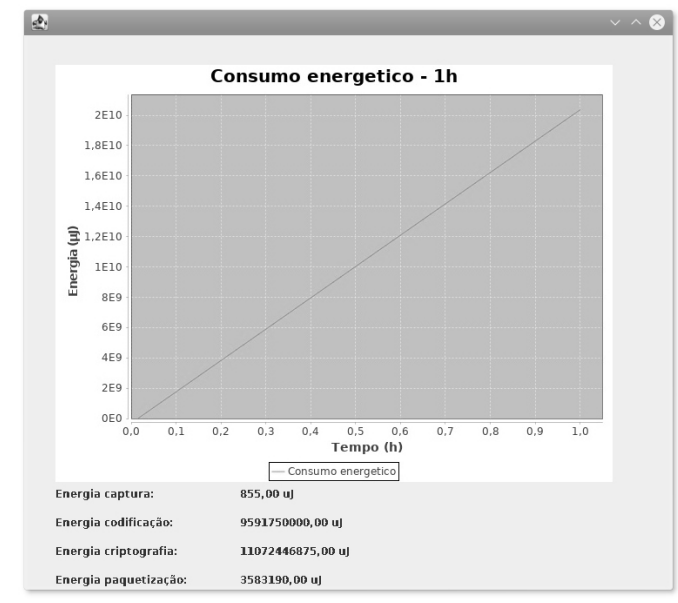

Figura 5: Resultados do teste com a ferramenta. 
Os resultados apresentados são uma primeira verificação em direção à utilização do modelo proposto como método efetivo de validação de redes de sensores visuais sem fio.

\subsection{Comparação com redes reais}

O segundo grupo de testes objetivou verificar a exatidão do modelo quanto a estimativa de consumo de energia. Para tal, implantou-se uma RSVSF, equipada com sensores Raspberry Pi 2, para realizar capturas de imagens de forma periódica.

Ao todo, foram utilizados 3 hardwares durante a análise de consumo de energia em laboratório, que são eles:

- Raspberry Pi 2: Plataforma central que executava os testes requeridos

- Arduíno Uno: Recebia o comando do Raspberry que lia a medição de consumo

- INA219: Circuito sensor de corrente que media o consumo de energia no Raspberry

O processo de captura começa com a alimentação do Raspberry, utilizando um adaptador que converte a tensão da rede elétrica em uma saída regulada de $5 \mathrm{~V}$, que servirá de fonte para alimentá-lo. O fio positivo foi cortado fisicamente e conectados os pinos $\mathrm{V}_{\text {in }+}$ e $\mathrm{V}_{\text {in- }}$ do INA219. O INA219 foi conectado ao Arduíno pelo protocolo I2C.

As medições foram realizadas com o auxílio de scripts que possuíam 3 blocos distintos. O primeiro acionava o pino 8 do Raspberry (GPIO - General Purpose Input Output), o segundo bloco possuía os comandos essenciais para execução da tarefa a ser medida e o terceiro bloco desativava o pino 8 do Raspberry. O Arduíno se mantém "escutando" o pino 8 desde o momento que é identificado o nível lógico alto. Dessa forma, foi possível realizar as medições com maior exatidão, pois, se tinha o momento exato da execução das instruções desejadas. A Figura 6 mostra o esquema de ligação entre os blocos. Todas as medições seguiram esse mesmo processo.

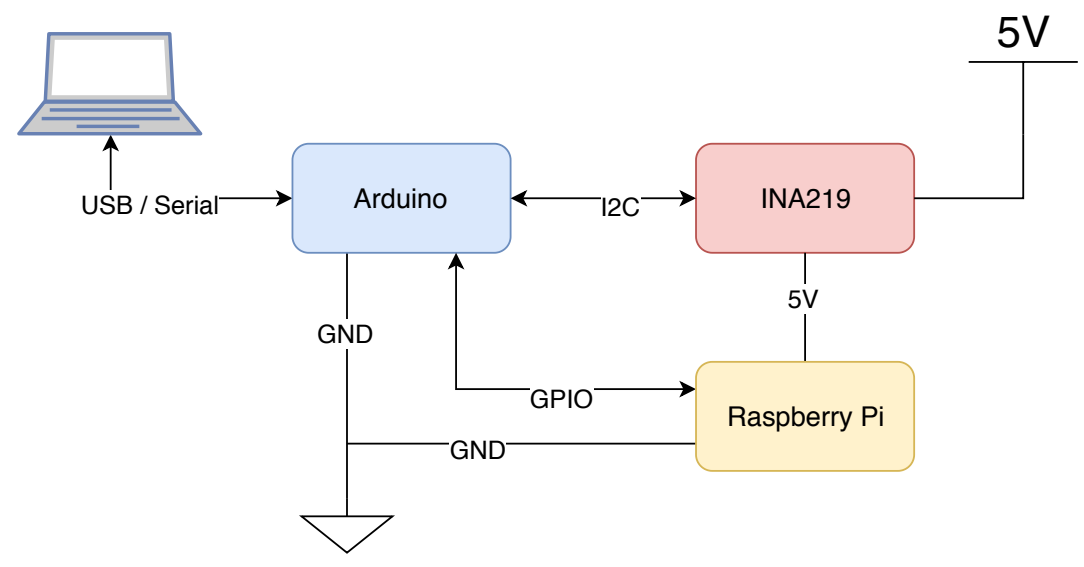

Figura 6: Esquema de hardwares. 
Inicialmente mediu-se quanto o Raspberry consumia estando em repouso. Verificou-se que ele possui uma potência média de 1,16W. Para as medições seguintes, esse valor foi descontado. As configurações utilizadas para a captura de imagem foram:

- Resolução de 2592 x 1942

- Imagens coloridas

- Sem compressão

Então, realizou-se uma captura de 30 imagens em sequência, sem que elas sejam salvas em disco, pois queria-se medir apenas o consumo proveniente da captura. Obteve-se um resultado de 1,89W. Descontando o valor do sistema em repouso, tem-se que a potência média do consumo de captura foi $0,73 \mathrm{~W}$. A captura dessas imagens durou um tempo de 99 segundos, podendo-se estimar um tempo médio de 3,3 segundos por imagem. Considerando que $\mathrm{W}$ é o mesmo que $\mathrm{J} / \mathrm{s}$, tem-se que o processo de captura de imagens consumiu 2,409J por imagem. O processo para medição do consumo de energia no processo de criptografia foi similar.

Em seguida, analisou-se o processo de criptografia. Para realizar essa medição, imagens (capturadas no processo anterior) são aplicadas ao algoritmo de criptografia AES-128, medindo assim o tempo e a potência média do processo. O procedimento foi repetido 30 vezes, tirando uma média dos resultados ao final. Obteve-se uma potência média de $2,21 \mathrm{~W}$ : descontando a potência de repouso, obtêm-se $1,05 \mathrm{~W}$ e um tempo de $0,977 \mathrm{~s}$. Com isso, calcula-se que o consumo de energia para criptografar uma imagem é de $1,016 \mathrm{~J}$.

Tabela 5: Medições de consumo do Raspberry Pi 2.

\begin{tabular}{cc}
\hline Módulo & Energia $(\mathbf{J})$ \\
\hline Captura & 2,409 \\
\hline Criptografia & 1,016 \\
\hline
\end{tabular}

Ao executar a ferramenta, ajustou-se o Módulo de Captura de forma a igualar as configurações já descritas, obtendo-se como resposta o valor de $2,168 \mathrm{~J}$ por captura realizada. Se comparado com o valor medido, atingiu-se uma exatidão de $90 \%$.

Ao criptografar imagens com o algoritmo AES-128, obteve-se como resposta o valor de 3,3J. Diferente do módulo de captura, ele atingiu um valor distante do calculado. Atribui-se essa diferença pela medição do consumo de energia das instruções elementares realizadas no Raspberry.

Para medir o consumo de energia das ações elementares do Raspberry Pi 2, foi utilizado um procedimento similar ao que foi empregado anteriormente, o qual utilizou códigos em linguagem C para construção dos scripts.

Os códigos binários dos scripts em linguagem C possuem operações de atribuição, adição e testes de condições, não sendo possível isolar as operações elementares desejadas. Essa problemática 
fez o consumo de energia aumentar, não representando o seu real consumo. As Tabelas 6 e 7 mostram os valores medidos.

Tabela 6: Consumo de energia de instruções do Raspberry Pi 2.

\begin{tabular}{ccccc}
\hline Ação & Quantidade & Consumo(W) & Tempo(s) & J/byte $(\mu J)$ \\
\hline Escrever flash & 104857 & 0,3307 & 9,011 & 28.419 \\
\hline Ler flash & 104857 & 0,266 & 0,241 & 0.611 \\
\hline
\end{tabular}

Tabela 7: Consumo de energia de instruções do Raspberry Pi 2.

\begin{tabular}{cllll}
\hline Ação & Quantidade & $\begin{array}{l}\text { Consumo } \\
(\mathbf{W})\end{array}$ & Tempo(s) & $\begin{array}{l}\text { J/instrução } \\
(\mu J)\end{array}$ \\
\hline Multiplicação & 1 bilhão & 0,244 & 5,596 & 0.00136 \\
\hline Adição & 1 bilhão & 0,290 & 4,496 & 0.00130 \\
\hline Divisão & 1 bilhão & 0,270 & 20 & 0.0054 \\
\hline Round & 100 milhões & 0,275 & 4,258 & 0.0117 \\
\hline Troca de bits & 1 bilhão & 0,318 & 3,408 & 0.00108 \\
\hline Or & 1 bilhão & 0,329 & 3,377 & 0.00111 \\
\hline And & 1 bilhão & 0,316 & 3,456 & 0.00109 \\
\hline
\end{tabular}

\section{CONCLUSÃO}

As redes de sensores se tornaram uma grande linha de pesquisa na Computação, muito devido à sua versatilidade e ao baixo custo de implantação. Como aplicações diferentes trazem desafios diferentes, soluções otimizadas são pensadas para cada caso, podendo ser expandidas em várias outras. A energia residual nas baterias dos dispositivos é um dos pontos críticos para a permanência ativa da rede, considerando que eles não estão ligados a uma fonte de energia permanente. Logo, pode-se dizer que essa linha de pesquisa ainda apresenta importantes desafios para serem tratados.

Neste artigo foi proposto um modelo matemático para estimativas de consumo de energia para nós sensores visuais sem fio. Para a realização dos testes, foi desenvolvida uma ferramenta de simulação que permite alterar parâmetros de configuração, possibilitando a realização de testes rápidos e confiáveis.

O modelo se mostrou preciso quanto a proximidade de suas respostas, se comparado com uma situação real. A única ressalva é a metodologia para descobrir o consumo de energia em instruções elementares do sensor utilizado.

Os trabalhos futuros estão focados na continuidade do modelo proposto e da ferramenta desenvolvida. Planeja-se ainda adicionar ao modelo a estimativa do consumo de energia para nós 
intermediários e nós sinks, considerando o consumo de energia no processo de transmissão de pacotes.

\title{
AGRADECIMENTOS
}

Os autores agradecem o suporte da FAPESB para a realização desta pesquisa.

\begin{abstract}
Wireless sensor networks have an important role in the current scenario of new communication technologies, focused on Internet of Things and Smart Cities applications. In those networks, energy issues are central, since many wireless sensor networks are developed to operate with battery-operated sensors. In such way, the proper planning of wireless sensor networks is required for good performance and there are some techniques to assess the performance of those networks without requiring deployment of real sensors. This article proposes a new mathematical model to assess energy consumption in camera-enabled sensors, allowing relevant measurements before deployment of physical networks. Besides this innovate mathematical model, a new tool was developed, referred as EnergyWVSN, aimed at the support of practical usage of the proposed model.
\end{abstract}

Keywords: wireless visual sensor networks, mathematical modelling, energy consumption, performance assessment.

\section{REFERÊNCIAS}

[1] M.A. Azgomi \& A. Khalili. Performance evaluation of sensor medium access control protocol using coloured petri nets. Electronic Notes in Theoretical Computer Science, 242(2) (2009), 31-42.

[2] P. Baldan, N. Cocco, A. Marin \& M. Simeoni. Petri nets for modelling metabolic pathways: a survey. Natural Computing, 9(4) (2010), 955-989.

[3] D.G. Costa, M. Collotta, G. Pau \& C. Duran-Faundez. A Fuzzy-Based Approach for Sensing, Coding and Transmission Configuration of Visual Sensors in Smart City Applications. Sensors, (2017), 1-17.

[4] D.G. Costa \& L.A. Guedes. Exploiting the sensing relevancies of source nodes for optimizations in visual sensor networks. Multimedia Tools and Applications, 64(3) (2013), 549-579. doi:10.1007/ s11042-011-0961-4. URL https://doi.org/10.1007/s11042-011-0961-4.

[5] D.G. Costa, L.A. Guedes, F. Vasques \& P. Portugal. Um Protocolo Genérico Eficiente de Energia para Aplicações em Redes de Sensores sem Fio sem Restrição de Tempo de Resposta. Revista de Tecnologia da Informação e Comunicação, (2015).

[6] N. Costa, J. Silva \& J.L. Silva. Real-time app development approach for indoor monitoring. In "Information Systems and Technologies (CISTI), 2017 12th Iberian Conference on”. IEEE (2017), pp. $1-4$.

[7] M.R. Doomun, K.S. Soyjaudah \& D. Bundhoo. Energy consumption and computational analysis of Rijndael-AES. In "Internet, 2007. ICI 2007. 3rd IEEE/IFIP International Conference in Central Asia on”. IEEE (2007), pp. 1-6. 
[8] M. Elhoseny, X. Yuan, Z. Yu, C. Mao, H.K. El-Minir \& A.M. Riad. Balancing energy consumption in heterogeneous wireless sensor networks using genetic algorithm. IEEE Communications Letters, 19(12) (2015), 2194-2197.

[9] G. Han, L. Liu, J. Jiang, L. Shu \& G. Hancke. Analysis of energy-efficient connected target coverage algorithms for industrial wireless sensor networks. IEEE Transactions on Industrial Informatics, 13(1) (2017), 135-143.

[10] B. Lacerda \& P.U. Lima. Petri nets as an analysis tool for data flow in wireless sensor networks. In "1st Portuguese Conference on WSNs, Coimbra, Portugal" (2011), pp. 1-6.

[11] V. Lecuire, C. Duran-Faundez \& N. Krommenacker. Energy-efficient transmission of wavelet-based images in wireless sensor networks. Journal on Image and Video Processing, 2007(1) (2007), 15-15.

[12] V. Lecuire, C. Duran-Faundez \& N. Krommenacker. Energy-efficient image transmission in sensor networks. International Journal of Sensor Networks, 4(1-2) (2008), 37-47.

[13] J.S. Leu, T.H. Chiang, M.C. Yu \& K.W. Su. Energy efficient clustering scheme for prolonging the lifetime of wireless sensor network with isolated nodes. IEEE communications letters, 19(2) (2015), $259-262$.

[14] P.H.F. Machado \& L. Souza. Proposiçao e modelagem de uma rede de sensores sem fio de baixo custo. XI Simpósio Brasileiro de Automaçao Inteligente, (2013), 1-6.

[15] A. Mammeri, A. Khoumsi, D. Ziou \& B. Hadjou. Modeling and adapting JPEG to the energy requirements of VSN. In "Computer Communications and Networks, 2008. ICCCN'08. Proceedings of 17th International Conference on”. IEEE (2008), pp. 1-6.

[16] L. Mokdad, J. Ben-Othman, B. Yahya \& S. Niagne. Performance evaluation tools for QoS MAC protocol for wireless sensor networks. Ad Hoc Networks, 12 (2014), 86-99.

[17] J.H.B. Neto, J.C. Júnior \& L.S. Rocha. A New Flow Network Approach for Improving Clustering Protocols in Wireless Sensor Networks. In "Advanced Information Networking and Applications (AINA), 2017 IEEE 31st International Conference on”. IEEE (2017), pp. 285-291.

[18] R. Neto, O. de Araújo et al. "Estudo e implementação de ip-cores para Criptografia simétrica baseada no Advanced encryption standard (AES)". Master's thesis, Universidade Federal da Paraíba (2013).

[19] C. Perera, A. Zaslavsky, P. Christen \& D. Georgakopoulos. Sensing as a service model for smart cities supported by internet of things. Transactions on Emerging Telecommunications Technologies, 25(1) (2014), 81-93.

[20] A. Shareef \& Y. Zhu. Energy modeling of processors in wireless sensor networks based on petri nets. In "Parallel Processing-Workshops, 2008. ICPP-W'08. International Conference on". IEEE (2008), pp. 129-134.

[21] A. Shareef \& Y. Zhu. Energy modeling of wireless sensor nodes based on Petri nets. In "2010 39th International Conference on Parallel Processing”. IEEE (2010), pp. 101-110.

[22] K. Su, J. Li \& H. Fu. Smart city and the applications. In "Electronics, Communications and Control (ICECC), 2011 International Conference on”. IEEE (2011), pp. 1028-1031. 
[23] E. Sun, X. Shen \& H. Chen. A low energy image compression and transmission in wireless multimedia sensor networks. Procedia Engineering, 15 (2011), 3604-3610.

[24] M.B.M. Taj \& M.A. Kbir. The impact of MAC protocols in energy consumption of transferring multimedia contents using Castalia simulator. 2nd International Conference on Electrical and Information Technologies ICEIT'2016, (2016), 521-525.

[25] A.S. Wander, N. Gura, H. Eberle, V. Gupta \& S.C. Shantz. Energy analysis of public-key cryptography for wireless sensor networks. In "Pervasive Computing and Communications, 2005. PerCom 2005. Third IEEE International Conference on”. IEEE (2005), pp. 324-328.

[26] F.G.H. Yap \& H.H. Yen. A Survey on Sensor Coverage and Visual Data Capturing/Processing/Transmission in Wireless Visual Sensor Networks. Sensors, 14(2) (2014), 3506-3527. doi:10.3390/s140203506. URL http: //www. mdpi . com/1424-8220/14/2/3506.

[27] J. Yick, B. Mukherjee \& D. Ghosal. Wireless sensor network survey. Computer networks, 52(12) (2008), 2292-2330.

[28] H. Zhang, S. Zhang \& W. Bu. A clustering routing protocol for energy balance of wireless sensor network based on simulated annealing and genetic algorithm. International Journal of Hybrid Information Technology, 7(2) (2014), 71-82. 